\title{
CHINA, UN DILEMA DE POLÍTICA EXTERIOR IRRESUELTO PARA AUSTRALIA
}

\section{Patricio Giusto*}

Director Ejecutivo del Observatorio SinoArgentino (Fundación Nuevas Generaciones)

$\triangle$ pgiusto@observatoriosinoargentino.com
Recibido: 10 de diciembre de 2019

Aceptado: 6 de febrero de 2020

DOI: $10.46553 /$ colec.31.1.2020.p53-68

Resumen: El presente artículo tiene por objeto analizar la estrategia de política exterior de Australia frente al ascenso económico de China, con foco en la última etapa de gobierno laborista (2007-2013) y la actual de la Coalición Liberal-Nacional (2013 a la fecha). China se ha consolidado como el principal socio comercial de Australia y una de sus principales fuentes de inversiones extranjeras directas. Al mismo tiempo, han sido constantes las controversias por la supuesta injerencia de China en los asuntos internos australianos. Ambos factores tensionaron la relación con los Estados Unidos, socio estratégico de Australia en defensa y seguridad. Ni la Coalición Liberal-Nacional ni el Partido Laborista han podido resolver el dilema que representa en materia de política exterior la creciente e insustituible dependencia económica con China. Pese a ser Australia una potencia media en términos económicos y militares, resulta llamativa su incapacidad para resolver

*El autor es candidato doctoral en Estudios Internacionales (Universidad Di Tella). Master of China Studies (Zhejiang University) y Magíster en Políticas Públicas (FLACSO). Lic. en Ciencias Políticas de la Pontificia Universidad Católica Argentina, donde es docente regular en varias materias en carreras de grado y en el Programa Ejecutivo sobre China Contemporánea. También es profesor visitante en Zhejiang University. En el Consejo Argentino para las Relaciones Internacionales coordina el Grupo de Trabajo sobre Australasia y es miembro de los grupos sobre China y Estados Unidos. Además, es director del Observatorio Sino-Argentino de la Fundación Nuevas Generaciones y de la consultora Diagnóstico Político. Participa asiduamente en conferencias y en medios de comunicación nacionales e internacionales. 
satisfactoriamente esta disyuntiva. En base a documentos oficiales y a fuentes bibliográficas y periodísticas especializadas, se aportan ideas preliminares al respecto y se compara el caso australiano con la similar situación que afronta Latinoamérica.

Palabras clave: Australia; China; Estados Unidos; Latinoamérica; política exterior

\begin{abstract}
The purpose of this article is to analyze Australia's foreign policy strategy in front of China's economic rise, focusing on the last period of Labor government (2007-2013) and the current government of the Liberal-National Coalition (2013 to date). China has become Australia's most important trading partner and one of its main sources of foreign direct investment. At the same time, controversy over China's alleged interference in Australian internal affairs has been remarkable. Both factors put a strain on the relationship with the United States, Australia's key strategic partner in defense and security. Neither the LiberalNational Coalition nor the Labor Party have been able to resolve the dilemma that the economic dependence with China and its interference represents for Australia's foreign policy. Despite being Australia a middle power, its inability to satisfactorily resolve this dilemma is striking. Based on official documents and specialized bibliographical sources, some preliminary ideas are provided in this regard, as well as a comparison between the Australian case and the similar situation facing Latin America.
\end{abstract}

Keywords: Australia; China; United States; Latin America; Foreign Policy

\title{
I. Introducción
}

Australia es el sexto país más grande del mundo, siendo a su vez la isla más grande del mundo, situada entre los océanos Índico y Pacífico. Apenas 25 millones de personas habitan un territorio de 7,7 millones de $\mathrm{Km} 2$, signado por una geografía adversa. El territorio marítimo circundante llega a 12 millones de $\mathrm{Km} 2$, una de las mayores masas de agua del planeta.

Por otra parte, Australia es una de las economías más estables, pujantes y abiertas del planeta (13 PBI global en 2017 [Banco Mundial 2017]). En cuanto a su sistema político, Australia es un Estado federal que adoptó la 
monarquía constitucional parlamentaria como forma de gobierno, tras lograr su independencia del Reino Unido, en 1901. Durante los últimos 100 años, liberales y laboristas han alternado el gobierno de una de las democracias más sólidas del mundo (Sutherland 2008).

Siendo una de las principales potencias medias a nivel global en términos económicos y militares, Australia se encuentra ahora ante una compleja disyuntiva, a raíz del imparable ascenso de China como superpotencia en la región del Indo-Pacífico. Históricamente, Australia mantuvo una sólida alianza militar y económica con los Estados Unidos (EE.UU.), potencia dominante en la región, desde el fin de la Segunda Guerra Mundial. No obstante, en las últimas décadas, China ha acrecentado notablemente su influencia económica y expansión marítima en la región, dejando a Australia en una situación geopolítica muy delicada (Wesley 2011).

China se ha convertido en el principal destino de las exportaciones australianas, al tiempo que la ex colonia británica también se ha beneficiado de los fenomenales flujos de inversiones del gigante comunista. Pero ello no ha sido gratuito para Australia: el fuerte avance de China vino acompañado de escándalos de espionaje e interferencia en la política doméstica. El principal afectado fue el Partido Laborista, con una visión más positiva respecto a la relación con China, desde la centro-izquierda. No obstante, la centro-derecha gobernante de la Coalición Liberal-Nacional (en adelante "Coalición"), no ha exhibido una política exterior consistente para afrontar el desafío de política exterior que supone esta compleja relación (Wang 2016).

Australia está frente a un dilema estratégico que permanece irresuelto. El mismo podría resumirse en la siguiente pregunta: ¿Cómo conciliar la creciente dependencia económica respecto a China con el vínculo estratégico e intereses geopolíticos compartidos con los EE.UU. en la región? ¿Qué factores han influido en la política exterior de Australia frente a China? Se trata de un caso de estudio con muchas enseñanzas para Latinoamérica, región cuyos países afrontan un dilema similar, salvando las enormes distancias geopolíticas y económicas. 


\section{Antecedentes de las relaciones entre China y Australia}

Los primeros contactos entre chinos y australianos se remontan a la "fiebre del oro" de fines del Siglo XVIII, cuando cientos de chinos, provenientes principalmente de Cantón, llegaron a la isla. A pesar de la larga historia de intercambios y la considerable población china que se fue asentando en Australia, la primera misión diplomática oriental en suelo australiano data de 1909. Dos años más tarde, tras la caída de la última dinastía imperial Qing, China entró en un largo período de convulsión interna y creciente aislamiento internacional (Wang 2016).

China y Australia establecieron relaciones diplomáticas oficiales recién en 1941. Tras el fin de la Guerra Civil China, en 1949, Australia pasó a reconocer al gobierno de los derrotados nacionalistas del Guomindang, que se refugiaron en la isla de Taiwán. En 1972, luego de la célebre visita del presidente estadounidense Richard Nixon a la República Popular China, Australia siguió el mismo camino de normalización de relaciones con el gobierno comunista.

Con el proceso de Reforma y Apertura chino, iniciado en 1978 por Deng Xiaoping, se inició una era dorada de relaciones entre China y Australia, favorecida por la cercanía geográfica y la enorme complementariedad en materia económica. Desde entonces, el gran motor de las relaciones económicas ha sido el insaciable apetito de China por los recursos naturales australianos, especialmente minerales. En las décadas del 80' y 90', compañías australianas comenzaron a invertir en sectores estratégicos de la economía china (Sherlock 1997).

Más recientemente, han sido las mega-empresas estatales chinas las que comenzaron a invertir fuertemente en minería, energía e infraestructura en Australia, al tiempo que las empresas de la isla siguen teniendo una presencia preponderante en la economía china. Sin dudas, un hito muy relevante de las relaciones económicas bilaterales se dio en 2015, cuando tras más de una década de negociaciones ambos países firmaron un Tratado de Libre Comercio (TLC).

Entre 2015 y 2018, las exportaciones de Australia a China pasaron de US\$ 78.000 millones a US\$ 118.000 (más del 30\% del total), con un abultado superávit comercial favorable a Australia, en el orden de los US\$ 44.000 millones para 2018. En materia de inversiones, empresas 
australianas invirtieron US\$ 75.300 millones en China, durante 2018. En tanto, empresas chinas hicieron lo propio en Australia por US\$ 63.500 millones (Australian Government 2019).

Las relaciones sino-australianas también fueron creciendo sostenidamente en otros planos, como ser los intercambios educativos y culturales. Un reciente informe estimó que uno de cada diez estudiantes universitarios en Australia es de nacionalidad china (alrededor de 200.000). Paradojalmente, al igual que sucede con el comercio exterior, esto ha sido un tema de controversia por la excesiva dependencia económica de las principales universidades australianas respecto a su matrícula de estudiantes chinos. Al mismo tiempo, unos 5.000 australianos se encuentran estudiando en universidades chinas (Babones 2019).

\section{Las relaciones sino-australianas durante el último período laborista}

En el año 2007, el laborista Kevin Rudd asumió como primer ministro de Australia, poniendo fin a once años de gobiernos de la Coalición liberal. Además de contar con una extensa trayectoria política, Rudd también era conocido como uno de los más prominentes sinólogos del mundo. Como era de esperarse, Rudd hizo un gran esfuerzo durante su gestión para promover buenas relaciones con China, desde una retórica pragmática; enfocado en seguir afianzando los vínculos económicos con la ascendente potencia asiática (Rudd 2018).

En una recordada gira oficial que Rudd hizo a Beijing en 2008, el australiano sorprendió al dar un discurso en chino, urgiendo a China a contribuir a la construcción de un "mundo armonioso" y convertirse en un "actor global responsable" (ACCW 2016a), replicando la retórica comunista. Rudd, figura muy elogiada por la prensa y el establishment político chino, sugirió en aquel discurso que Australia debía explorar una "tercera vía" para relacionarse con China, "entre el conflicto y el kowtow" (acto ritual de inclinarse frente al emperador). Dicho enfoque, que sonaba lógico y realista, fue bien recibido incluso por los chinos (ACCW 2016a).

Este momentum en las relaciones sino-australianas se vio repentinamente opacado por el primer gran escándalo en materia de interferencia política y espionaje. En julio de 2009, cuatro empleados de la minera australiana Rio 
Tinto, sindicados de corrupción y espionaje industrial, fueron condenados a prisión en Shanghái. Stern Hu, uno de los australianos imputados reconoció haber cobrado US\$ 900.000 en sobornos. Las penas fueron consideradas "severas" por el gobierno de Australia, denegado de proveer asistencia consular durante el juicio. ${ }^{1}$

El caso tuvo fuerte repercusión mediática en ambos países e impactó sobre las relaciones diplomáticas entre China y Australia, generando un clima de mutua desconfianza. Ese mismo año, Australia difundió un libro blanco de defensa calificando la expansión militar de China en el Pacífico como una "causa de preocupación" para su seguridad (Australian Government 2009). El escándalo de Río Tinto, sumado a otras cuestiones más vinculadas a la política doméstica, precipitaron el fin del primer gobierno de Rudd.

En 2010, lo laboristas lograron retener el poder de la mano de Julia Gilliard, quien asumió con una visión de política exterior fuertemente orientada hacia Asia y con la clara intención de recomponer y expandir las relaciones con China. En octubre de 2012, el gobierno difundió el libro blanco sobre "Australia en el Siglo de Asia", destacando la importancia de China, India y otros países de la región para el futuro de Australia (Australian Government 2012).

En 2013, tras una exitosa gira de Gilliard a Beijing, Australia y China firmaron una "asociación estratégica", logro elogiado incluso por los liberales. ${ }^{2}$ Gilliard perdió apoyo partidario y fue reemplazada ese año por Rudd, quien tuvo un segundo mandato como primer ministro que duró apenas 83 días. De este breve período se destaca el esfuerzo de Rudd por acelerar las estancadas negociaciones por un acuerdo de libre comercio con China, el cual fue colocado como una de sus prioridades de política exterior (Wang 2016).

1 "Rio Tinto accused of massive fraud and espionage in China". Artículo publicado en Business Insider el 10-Ago-2009: https://www.businessinsider.com/rio-tinto-accusedof-massive-fraud-and-espionage-in-china

2 "Gillard scores coup with China agreement". Artículo publicado en The Sidney Morning Herald el 10-Abr-2013: https://www.smh.com.au/politics/federal/gillardscores-coup-with-china-agreement-20130409-2hjin.html 


\section{La oscilante relación con China durante el gobierno de Abbott}

En septiembre de 2013, la Coalición retomó el poder en Australia, de la mano de Tony Abbott. A diferencia de la agenda de los laboristas, China no aparecía entre los temas más relevantes en materia de política exterior para los liberales, al menos durante la campaña. No obstante, Abbott ya exhibía diferencias notables con sus antecesores, como ser su oposición a la inversión extranjera directa de empresas extranjeras para adquirir grandes extensiones de tierra y explotar recursos naturales en Australia (ACCW 2016b).

Una vez en el poder, las relaciones bilaterales ingresaron rápidamente en una zona de turbulencia: Abbott sorprendió al anunciar su intención de ampliar las relaciones diplomáticas y comerciales con Taiwán, en línea con un mayor acercamiento a los EE.UU. en la región. De hecho, estas declaraciones fueron seguidas de un relanzamiento de la plataforma de "diálogo trilateral" y realización de ejercicios militares, junto a Japón y los EE.UU. (Wang 2016).

Por otra parte, Abbott cumplió su compromiso de reducir el umbral para las inversiones extranjeras directas en adquisición de tierras y explotación de recursos naturales, afectando fuertemente a inversores chinos. No obstante, en su primer encuentro con el flamante mandatario chino $\mathrm{Xi}$ Jinping, en octubre de 2013, Abbott se comprometió a acelerar las negociaciones por el TLC. Algunos analistas lo percibieron como una contradicción. ${ }^{3}$

La tensión con China tuvo una nueva escalada en noviembre de ese año, tras la decisión de Abbott de prohibir el ingreso de la compañía china Huawei a la red nacional de telecomunicaciones, en base a un crítico informe sobre los riesgos que ello implicaría elaborado por la agencia australiana de inteligencia (ASIO). Esta medida fue criticada en duros términos desde Beijing (ACCW 2016b). La escalada pasó a un nuevo nivel con la decisión china de crear una "zona defensiva de identificación aérea"

\footnotetext{
3 "Tony Abbott vows to strengthen trade with China after meeting Xi Jinping on sidelines of APEC'. Artículo publicado en $A B C$ News el 6-Oct-2013: https:/www.abc.net.au/news/2013-10-07/abbott-meets-chinese-president-onsidelines-of-apec/5001922
} 
en el Mar del Este de China, sobre territorios disputados con Japón. Australia fue el único país en convocar al embajador chino para pedir explicaciones. ${ }^{4}$ China calificó de "completamente equivocada" la postura australiana y Abbott redobló la apuesta, buscando incrementar los vínculos con Japón y los EE.UU. (ACCW 2016b).

Hacia inicios de 2014, Abbott advirtió la necesidad de bajar el nivel de tensión con China y se embarcó en una gira oficial hacia Beijing con más de 600 referentes políticos y empresariales australianos, buscando poner la “diplomacia económica" por encima de la política y los valores. A pesar del pesimismo que imperaba previo a la gira, la misma fue muy satisfactoria. Se firmaron numerosos acuerdos y las relaciones entraron en una saludable etapa de distención. Abbott le expresó a Xi Jinping el deseo australiano de ser un "verdadero amigo" de China y "ayudar a construir el siglo asiático", en sintonía con la visión de los laboristas. ${ }^{5}$

En noviembre de 2014, el presidente Xi visitó Australia para la cumbre del G20. Fue otro avance en las relaciones. Ambos países actualizaron su status, pasando a una "asociación estratégica integral". Además, se firmó finalmente el demorado TLC. De todas formas, Abbott se mostró cauto frente a la invitación de Xi a adherir a la mega-iniciativa china denominada Belt and Road Initiative (BRI) e integrarse al nuevo Banco Asiático de Inversión e Infraestructura (AIIB). ${ }^{6}$ No obstante, en marzo de 2015 Australia ingresó a dicho banco.

Privilegiando valores e intereses geopolíticos compartidos, Abbott buscó ensayar una política exterior mucho más cercana a los EE.UU. y Japón, tratando de disociar ese giro de la floreciente relación comercial con China.

4 “Tony Abbott says we'll 'speak our mind' on China's new air-defence zone". Artículo publicado en The Guardian el 28-Nov-2013:

https://www.theguardian.com/world/2013/nov/28/tony-abbott-says-well-speak-ourmind-on-chinas-new-air-defence-zone

5 "Prime Minister Tony Abbott meets with China's president Xi Jinping in Beijing". Artículo publicado en $A B C$ News el 11-Abr-2014: https://www.abc.net.au/news/201404-11/tony-abbott-meets-chinese-president-xi-jinping/5385454

6 "China and Australia sign historic free trade agreement". Artículo publicado en The Guardian el 17-Nov-2014:

https://www.theguardian.com/australia-news/live/2014/nov/17/g20-brisbane-wrapsup-as-tony-abbot 
En parte, puede decirse que fue exitoso en su cometido. De todas formas, Australia se mantuvo sin una política exterior clara frente al ascenso de China y no resolvió la creciente dependencia en términos económicos (Walker 2018).

\section{El deterioro de las relaciones sino-australianas con Turnbull y Morrison}

En septiembre de 2015, el liberal Malcolm Turnbull sucedió a Tony Abbott como primer ministro, tras arrebatarle el liderazgo de la Coalición. Durante este período, las relaciones sino-australianas se sumergieron en uno de sus puntos más bajos en años (Walker 2018).

Paradojalmente, la era de Turnbull inició con un histórico hito en las relaciones con China: En diciembre de 2015 entró en vigencia el tratado de libre comercio firmado por Abbott. ${ }^{7}$ No obstante, Turnbull tomó la decisión de profundizar el acercamiento con los EE.UU., expandiendo la cooperación militar y escalando la retórica contra la expansión naval china en los mares del Este y Sur de China. Uno de los mayores momentos de tensión diplomática se dio en julio de 2016, cuando la Corte Internacional de La Haya falló contra China en sus disputas territoriales con Filipinas. Australia firmó un comunicado conjunto con Japón y los EE.UU., instando a China a respetar la decisión del tribunal, cosa que China repudió. ${ }^{8}$

Ese mismo año se relanzó el foro informal de "Cooperación Cuadrilateral”, compuesto por EE.UU., India, Australia y Japón, en clara oposición a China y su proyección en la región. Tras desembarcar sorpresivamente en la Casa Blanca, a fines de 2016, Donald Trump celebró y apoyó la política exterior australiana, cada vez más hostil hacia China (Wang 2016).

\footnotetext{
7 Australian Trade and Investment Comission (2015). "China-Australia Free Trade Agreement" (ChAFTA): https:/www.austrade.gov.au/australian/export/free-tradeagreements/chafta

8 "Julie Bishop stands by South China Sea stance after sharp criticism from China". Artículo publicado en The Guardian el 14-Jul-2016:

https://www.theguardian.com/world/2016/jul/15/julie-bishop-south-china-seacriticism-china
} 
En junio de 2017, Turnbull lanzó una nueva embestida diplomática contra China, al anunciar un amplio estudio sobre espionaje e interferencia política extranjera en Australia. Fue tras conocerse un reporte periodístico sobre supuestos intentos chinos de influir en políticos australianos y ejercer presión sobre los estudiantes internacionales en Australia. ${ }^{9}$

La postura de Turnbull fue anticipatoria del huracán por venir. En diciembre de ese año, el senador laborista Sam Dastyari renunció a su banca luego de contradecir la política de su partido respecto a China y de ser acusado de recibir sobornos de empresas chinas. El caso derivó en un escándalo político y mediático de grandes proporciones, que golpeó duro a los laboristas y, al mismo tiempo, envalentonó a Turnbull y a los halcones liberales anti-China. ${ }^{10}$

En medio del escándalo por el caso Dastyari, Turnbull declaró que los australianos se "levantarán" frente a la interferencia de China. La asociación con el slogan comunista enarbolado por Mao Zedong, en 1949, fue inmediata. Los dichos del primer ministro australiano fueron calificados por Beijing como "increíbles" y "ofensivos". ${ }^{11}$ Poco después, Turnbull logró amplio consenso político para avanzar en una serie de regulaciones que prohibieron las donaciones extranjeras a partidos políticos y grupos de activistas australianos. ${ }^{12}$ Fue un giro impactante, ya que Australia jamás tuvo ese tipo de restricciones, siendo uno de los países más abiertos y transparentes del mundo. Al mismo tiempo, Turnbull mantuvo el bloqueo total a Huawei para operar las futuras redes de 5G (en soledad con Japón y

9 "Malcolm Turnbull orders inquiry following revelations ASIO warned parties about Chinese donations". Artículo publicado en $A B C$ News el 6-Jun-2017: https:/www.abc.net.au/news/2017-06-06/turnbull-orders-inquiry-followingrevelation-asio-warned-parties/8592308

10 "Sam Dastyari quits as Labor senator over China connections". Artículo publicado en The Guardian el 11-Dic-2017: https:/www.theguardian.com/australianews/2017/dec/12/sam-dastyari-quits-labor-senator-china-connections

11 “Turnbull says Australia will 'stand up' to China”. Artículo publicado en South China Morning Post el 19-Dic-2017:

https:/www.scmp.com/news/asia/australasia/article/2123617/turnbull-says-australiawill-stand-china-foreign-influence-row

12 “Australia passes foreign interference laws amid China tension". Artículo publicado en $B B C$ News el 28-Jun-2018: https:/www.bbc.com/news/world-australia-44624270 
los EE.UU. $)^{13}$, como así también la negativa australiana de adherir a la BRI (Tobin 2019).

En agosto de 2018, el liberal Scott Morrison sucedió a Turnbull en el gobierno australiano. El corto período que lleva Morrison en el poder lejos estuvo de aportar paños fríos y claridad estratégica en las relaciones con China. Además de confirmar la línea de mayor alineamiento geopolítico con los EE.UU. y elevar el criticismo frente a China ${ }^{14}$, con Morrison resurgió con fuerza la cuestión de los derechos humanos, complicando aún más los vínculos diplomáticos. En julio de 2019, Australia firmó junto a otros 22 países una dura carta contra los supuestos abusos de las autoridades chinas en la región autónoma Uigur de Xinjiang. ${ }^{15}$

Para colmo, con Morrison se agudizó la controversia por la interferencia política de China, con nuevos casos de espionaje que resonaron fuerte en los medios. En noviembre, Morrison reconoció que Australia investigaba si China intentó infiltrar a un espía en su Parlamento. Grupos de la inteligencia china habrían ofrecido unos US\$ 800.000 a un australiano de origen chino para que se presentara como candidato del Partido Liberal por un escaño en el distrito de Melbourne. ${ }^{16}$ Este nuevo escándalo retroalimentó el sentimiento anti-China en los sectores más duros y nacionalistas de la Coalición gobernante, pero también volvió a exponer la incapacidad de los sucesivos gobiernos para gestionar de manera consistente la relación con

13 En 2019, Nueva Zelanda se sumó al lote de países con bloqueo total a Huawei. No obstante, Nueva Zelanda sí adhirió a la BRI ese mismo año. Otro pequeño lote de países europeos impuso restricciones parciales a Huawei, mientras que la gran mayoría de los países del mundo no impusieron ni planean ninguna restricción.

14 "Scott Morrison supports US push against China as Donald Trump touts 'unbreakable bond' with Australia". Artículo publicado en South China Morning Post el 21-Sep2019: https:/www.scmp.com/news/asia/australasia/article/3029781/scott-morrisonsupports-us-push-against-china

15 "22 countries sign letter calling on China to close Xinjiang Uyghur camps". Artículo publicado en $C N N$ el 11-Jul-2019: https:/edition.cnn.com/2019/07/11/asia/xinjianguyghur-un-letter-intl-hnk/index.html

16 “Australia investiga si China intentó infiltrar a un agente en su Parlamento". Artículo publicado en El País el 25-Nov-2019:

https://www.lavanguardia.com/internacional/20191125/471854117883/australiainvestiga-china-infiltrar-agente-parlamento.html 
China e impedir su supuesta interferencia en los asuntos internos (Walker 2018).

En ese sentido, el fracaso de Australia para lidiar con estas cuestiones convirtió al país en un caso paradigmático de la supuesta penetración ('sharp power') de China para manipular la política doméstica de sus principales socios (Clive 2018, Garnaut 2018 y Medcalf 2017). Este fenómeno se ha visto reflejado en dos populares series de Netflix: Pine Gap y Secret City. ${ }^{17}$

\section{Futuro incierto para el dilema australiano frente a China}

En base a lo analizado previamente, puede inferirse que laboristas y liberales han carecido de una política exterior consistente frente a China. La creciente dependencia económica de Australia con China, ha sido un factor decisivo, cuestión que se terminó imponiendo a las agendas de ambas coaliciones cuando les tocó gobernar. Los laboristas le asignaron mucha importancia a la relación con China, desde una óptica mayormente positiva sobre su ascenso y la importancia de la potencia asiática para el futuro de Australia. Con mucho pragmatismo, pusieron lo económico por encima de otros valores e intereses fundamentales para Australia. No obstante, se vieron duramente afectados por resonantes casos de interferencia y espionaje.

Por su parte, los liberales claramente ensayaron una posición más distante y confrontativa frente a China, al menos en el plano de los valores y la geopolítica. Este giro fue en favor de un mayor acercamiento con los EE.UU., históricamente el principal socio de Australia en materia de defensa y seguridad en la región, ahora enfrentado con China en el marco de una disputa geoestratégica. En lo económico, los liberales impusieron restricciones al ingreso de capitales y tecnología de China, amparados en la supuesta "interferencia extranjera", aunque buscando no afectar los

\footnotetext{
17 "Gracias al streaming, Australia quiere ser el centro de la ficción". Artículo publicado en La Nación el 4-Ene-2019: https://www.lanacion.com.ar/espectaculos/series-detv/gracias-al-streaming-australia-quiere-ser-el-centro-de-la-ficcion-nid2207733
} 
intercambios comerciales. De hecho, durante el gobierno liberal de Turnbull, en 2015, se concretó la demorada firma de un TLC con China.

La creciente ideologización de la política exterior australiana, sobre todo en el actual gobierno de Morrison, complicó aún más las oscilantes relaciones con China. A ello se sumaron nuevos casos de espionaje que constatan irresuelto desafío de seguridad que afronta Australia.

Está claro que China seguirá siendo el principal socio económico de Australia. Y es esperable esa dependencia siga en aumento. En ese marco, la guerra comercial y tecnológica entre China y los EE.UU. ha sido pésima para Australia, que ha quedado en una compleja situación geopolítica. Australia ha sido uno de los países más comprometidos por este conflicto (Geopolitical Futures 2018).

¿Es posible para una potencia media como Australia conciliar la fuerte relación económica con China y la sólida alianza militar con los EE.UU.? Ninguna de las dos relaciones parece reemplazable para Australia. Pero ni laboristas ni liberales han hallado una fórmula adecuada y sustentable para lograr un equilibrio entre ambas potencias. La pretendida "tercera vía" de Rudd se quedó a mitad de camino, mientras que la postura liberal ha estado plagada de contradicciones y posicionamientos ideológicos sin resultados claros (Henry 2019).

En ese sentido: ¿Ha sido Trump y su guerra tecno-comercial contra China la principal causa del agravamiento de las tensiones entre China y Australia? ¿O bien ello tiene más que ver con la política exterior poco consistente e ideologizada de Australia? Esto último ha sido especialmente notable en el actual gobierno de Morrison. Los derechos humanos se agregaron a la agenda de otros temas espinosos irresueltos, como la interferencia política y el espionaje.

Al mismo tiempo, cabe preguntarse: ¿Cómo seguirá el debate entre "duros" y "moderados" dentro de la Coalición gobernante? Morrison está claramente en el primer bando, fortalecido luego del triunfo electoral de mayo de 2019 que, de alguna forma, convalidó también su política exterior. Sin dudas, se trata de un escenario de suma incertidumbre para Australia, que deja numerosas enseñanzas para los países de Latinoamérica, plantados frente a un dilema similar. 


\section{Reflexión final: El dilema latinoamericano}

Si una potencia media y económicamente estable como es Australia, modelo en muchos aspectos a nivel global, no ha podido resolver satisfactoriamente el dilema que representa lograr un equilibrio entre China y los EE.UU., urge tomar dimensión del gigantesco desafío que implica esta misma cuestión para nuestra región. Latinoamérica está políticamente fragmentada, con procesos de ebullición social en curso y estancada económicamente. Para colmo, pareciera que hay tantas posturas sobre China como países hay en la región.

Mientras los EE.UU. han experimentado un cierto repliegue de Latinoamérica, posando su foco de atención en otras partes del mundo, presionan fuerte a los gobiernos de la región para que limiten su cooperación con China. Sobre todo, en áreas sensibles como tecnología y defensa.

Es un hecho que China ya es el primer o segundo socio comercial de los países de la región, sumado a la fuerte dependencia de algunas naciones como Argentina en materia de inversiones y finanzas, respecto a la potencia asiática. La región afronta un dilema similar al australiano, aunque a priori mucho más complejo de resolver, ya que nos encuentra mucho más vulnerables.

\section{Referencias}

Australian Centre on China in the World (ACCW). 2016a. "Kevin Rudd and Australia-China Relations". The Australia China-Story. Canberra: Australian National University.

. 2016b. "The Abbott Government and Australia-China Relations".

The Australia China-Story. Canberra: Australian National University.

Australian Government. 2009. Defending Australia in the Asia-Pacific Century: Force 2030. Canberra: Department of Defense.

2012. Australia in the Asian Century. Canberra: Department of the Prime Minister and Cabinet.

- 2019. A Safer Australia. A Secured Future. Our Plan to protect Australia and keep Australians safe. Canberra: Department of Defense. 
Babones, Salvatore. 2019. The China student boom and the risks it poses to Australian universities. New York: CIS (Centre for Independent Studies).

Banco Mundial. 2017. Datos de libre acceso del Banco Mundial. Disponible en: https://datos.bancomundial.org

Garnaut, John. 2018. "How China Interferes in Australia". Washington DC: Foreign Affairs (March $9^{\text {th) }}$.

Geopolitical Futures. 2018. Articulating Australia's Interests. Nueva York: Ediciones GP.

Hamilton, Clive. 2018. Silent Invasion: China's Influence in Australia. Sidney: Hardie Grant Books.

Henry, Ian. 2019. "In Australia's China 'debate', there is no monopoly on morality". The Interpreter (December $13^{\text {th }}$ ).

Medcalf, Rory. 2017. "China's influence in Australia is not ordinary soft power”. Australian Financial Review (June 7th).

Rudd, Kevin. 2018. The PM years. Canberra: Macmillan Australia.

Sherlock, Stephen. 1997. Australia's Relations with China: What's the Problem? Canberra: Foreign Affairs, Defense and Trade Group of the Parliament of Australia.

Sutherland, Alexander. 2008. History of Australia and New Zealand. Canberra: Public Domain.

Tobin, Meghan. 2019. "Huawei ban: Australia becomes increasingly isolated among Five Eyes". South China Morning Post (April $26^{\text {th }}$ ).

Walker, Tony. 2018. "Australia needs to reset the relationship with China and stay cool". The Conversation (june $3^{\text {rd }}$ ).

Wang, Yi. 2016. Australia-China relations post 1949. Sixty years of trade and politics. London: Taylor and Francis group.

Wesley, Michael. 2011. There Goes the Neighborhood: Australia and the Rise of Asia. Sidney: National University of Australia Editorial.

White, Hugh. 2013. "Australia's Choice". Foreign Affairs Magazine (September 3rd).

Medios periodísticos consultados

ABC News (España)

BBC News (Reino Unido) 
Business Insider (Estados Unidos)

CNN (Estados Unidos)

El País (España)

La Nación (Argentina)

The Guardian (Australia)

Sidney Morning Herald (Australia)

South China Morning Post (Hong Kong). 